\title{
The utility of $A D C$ parameters in the diagnosis of clinically significant prostate cancer by 3.0-Tesla diffusion-weighted magnetic resonance imaging
}

\author{
Aylin Altan Kus \\ Department of Radiology, Acibadem Mehmet Ali Aydinlar University, Atakent Hospital, İstanbul, Turkey
}

\begin{abstract}
Purpose: This study has focused on investigating the relationship between the exponential apparent diffusion coefficient (exp-ADC), selective apparent diffusion coefficient (sel-ADC) values, the ADC ratio (ADCr), and prostate cancer aggressiveness with transrectal ultrasound-guided prostate biopsy in patients with prostate cancer.

Material and methods: All patients underwent a multiparametric magnetic resonance imaging (mpMRI) including tri-planar T2-weighted (T2W), dynamic contrast-enhanced (DCE), diffusion-weighted sequences using a 3.0-Tesla MR scanner (Skyra, Siemens Medical Systems, Germany) with a dedicated 18-channel body coil and a spine coil underneath the pelvis, with the patient in the supine position. Exp-ADC, sel-ADC, and ADCr of defined lesions were evaluated using region-of-interest-based measurements. Exp-ADC, sel-ADC, and ADCr were correlated with the Gleason score obtained through transrectal ultrasound-guided biopsy.

Results: Patients were divided into 2 groups. Group I is Gleason score $\geq 3+4$, group II is Gleason score $=6$. Sel-ADC and exp-ADC were statistically significant between 2 groups ( 0.014 and 0.012 , respectively). However, the ADCr difference between nonclinical significant prostate cancer from clinically significant prostate cancer was not significant $(p=0.09)$.

Conclusions: This study is the first to evaluate exp-ADC and sel-ADC values of prostate carcinoma with ADCr. One limitation of this study might be the limited number of patients. Exp-ADC and sel-ADC values in prostate MRI imaging improved the specificity, accuracy, and area under the curve (AUC) for detecting clinically relevant prostate carcinoma. Adding exp-ADC and sel-ADC values to ADCr can be used to increase the diagnostic accuracy of DWI.
\end{abstract}

Key words: prostate cancer, Gleason score, diffusion-weighted imaging, multiparametric prostate MR, apparent diffusion coefficient.

\section{Introduction}

Prostate cancer (PCa) is the most common noncutaneous malignancy among Western men and the leading cause of cancer mortality, preceded only by lung cancer in the United States, with an annual incidence of 161,360 cases resulting in 26,730 deaths in 2018 [1]. PCa is most often diagnosed on systematic transrectal ultrasound (TRUS)guided prostate biopsy. TRUS-guided prostate biopsy is considered the gold standard for diagnosis of PCa, which should include 12 cores, thereby leading to a detection rate ranging between $24 \%$ and $44 \%$ according to the current European Association of Urology (EAU) guidelines.

It is crucial to diagnose tumour aggressiveness or, in other words, to correctly differentiate nonclinical significant prostate cancer (ncsPCa) from clinically significant prostate cancer (csPCa), to apply the appropriate treatment modalities and reduce overtreatment or ineffective treatment results $[2,3]$. CsPCa is defined as GS $\geq 3+4=7$, tumour measuring $\geq 0.5 \mathrm{~cm}^{3}$, or showing features of

Correspondence address:

Aylin Altan Kus, Department of Radiology, Acibadem Mehmet Ali Aydinlar University, Atakent Hospital, İstanbul, Turkey, e-mail: aylinaltan@gmail.com

Authors' contribution:

A Study design · B Data collection · C Statistical analysis · D Data interpretation - E Manuscript preparation · F Literature search · G Funds collection 
extraprostatic extension [4]. PCa is primarily based on prostate-specific antigen (PSA) screening and TRUS-guided prostate biopsy. However, prostatitis can also cause elevated PSA, and normal PSA does not exclude a tumour $[5,6]$.

Multiparametric magnetic resonance imaging (MpMRI) has assumed a primary role in the diagnosis of $\mathrm{PCa}$; it is non-invasive and the gold standard diagnostic imaging modality for $\mathrm{PCa}$, which is a combination of standard anatomical T1- and T2-weighted imaging with functional imaging techniques (diffusion-weighted imaging [DWI] and dynamic contrast-enhanced MRI). DWI measures the Brownian motion of water molecules in vivo on unenhanced MRI sequences and provides information about tissue cellularity and membrane integrity. It has shown potential to increase the accuracy of tumour detection, localization, and characterization of prostate cancer [7-10]. We can measure the water molecules' mobility (Brownian motion) in vivo on unenhanced MRI sequences and also provide apparent diffusion coefficient (ADC) map-derived DWI sequences, which are used to inversely correlate with cellularity in different tumour types, provides quantitative information about tumour aggressiveness, and has shown an overall high correlation with Gleason score (GS) $[11,12]$. However, in some reported studies, the ranges of ADC values have again demonstrated significant overlaps within the same GS groups $[13,14]$.

To avoid these inconsistencies and standardize the quantitative metrics, the ADC selective (sel-ADC), ADC exponential (exp-ADC), and $\mathrm{ADC}$ ratio (ADCr) (the quantitative parameter ratio between the tumour and nontumoural prostatic tissue) were also evaluated in this study. Several earlier studies demonstrated that the ADCr had better correlation with GS than the mean ADC value $[15,16]$ as well as a favourable impact on diagnostic performance [17].

This study aims to investigate the relationship between ADC parameters and Gleason score with the aid of histopathological findings by TRUS-guided prostate biopsy in patients with prostate cancer.

\section{Material and methods}

\section{Study population}

This retrospective study was approved by the responsible ethics committee. We performed a search using our institutional Picture Archiving and Data Reporting System (PACS; Centricity, General Electric CA, U.S.) to identify all patients who underwent multiparametric prostate MRI and TRUS-guided biopsy of the prostate between July 2016 and March 2019. Forty-four consecutive patients were retrospectively included in the study with the following including criteria: a) 3-Tesla MR platform with an 18-channel phased array surface coil and a spine coil underneath the pelvis; b) at least 1 suspicious lesion with a large axial diameter of $5 \mathrm{~mm}$ in the peripheral zone and a PIRADS-v2 score $>3$ - each identified lesion is scored according to the PIRADS v2 classification system from ESUR; c) each patient was diagnosed as prostatic adenocarcinoma histopathologically with TRUS biopsy using a Loqic E9 (General Electric Healthcare) PSA - PSA density levels and prostate volumes were recorded - TRUSguided prostate biopsy was performed involving 14 cores after the magnetic resonance imaging (MRI) examinations.

\section{Magnetic resonance imaging protocols and imaging analysis}

\section{Magnetic resonance imaging acquisition}

All patients underwent a multiparametric prostate MRI including tri-planar T2-weighted (T2W), dynamic contrastenhanced (DCE), diffusion-weighted imaging sequences using a 3.0-Tesla MR scanner (Skyra, Siemens Medical Systems, Germany) with a dedicated 18-channel body coil and a spine coil underneath the pelvis, with the patient in the supine position. Routinely, just before the beginning of the examination, $20 \mathrm{mg}$ butylscopolamine bromide (Buscopan, Boehringer Ingelheim, Germany) was injected (i.m.) to reduce bowel movements. $135 \mathrm{ml}$ sodium di-hydrogen phosphate + di-sodium hydrogen phosphate (B.T. enema) was administrated to the patient to reduce motion artifacts due to bowel peristalsis. We imaged the entire prostate and oriented axial images to be perpendicular to the rectal wall guided by sagittal images.

Prostate MRI was done using the following protocol - axial T2-weighted (T2W) sequence (TR/TE 3200/96 $\mathrm{ms}$; slice thickness $3.5 \mathrm{~mm}$ with $0.5 \mathrm{~mm}$ interslice gap; field of view [FOV] $200 \times 200 \mathrm{~mm}$; matrix size $448 \times 336$; average 2 ) sagittal T2W volumetric space sequence (TR/ TE $1500 / 139 \mathrm{~ms}$; slice thickness $0.9 \mathrm{~mm}$ without any interslice gap; FOV $220 \times 220 \mathrm{~mm}$; matrix size $256 \times 205$; average 1.4); a coronal T2W sequence (TR/TE 4870/117 $\mathrm{ms}$; slice thickness $3 \mathrm{~mm}$ with $0.6 \mathrm{~mm}$ interslice gap; FOV $240 \times 240$; matrix size $314 \times 448$; average 1 ) echo planar imaging (EPI)-based DWI sequence in axial planes at $b$ values of $50,400,1000,1400 \mathrm{~s} / \mathrm{mm}^{2}(\mathrm{TR} / \mathrm{TE} 4100 / 61 \mathrm{~ms}$; slice thickness $3.5 \mathrm{~mm}$; FOV $200 \times 200$, and matrix size $114 \times 114)$; an axial multiphase 3 -dimensional (3D) T1W dynamic gradient-echo sequence obtained after $10 \mathrm{ml}$ intravenous bolus injection of $0.1 \mathrm{mmol}$ gadobutrol (Gadovist; Bayer Laboratories, Berlin, Germany) per kilogram of body weight followed by a $20 \mathrm{ml}$ saline flush at a rate of $2.0 \mathrm{ml} / \mathrm{s}$. Approximately 32 series were obtained, which lasted for a total of 8 minutes with flip angle $15^{\circ}$; slice thickness $3.5 \mathrm{~mm}$ with $0.6 \mathrm{~mm}$ interslice gap; FOV $220 \times 220$; matrix $80 \times 192$, average $1 \mathrm{~mm}$.

Pelvic postcontrast T1 axial fat-saturated Turbo Spin Echo (TSE; TR/TE 515/20; slice thickness $5 \mathrm{~mm}$ with $1 \mathrm{~mm}$ interslice gap, FOV $350 \times 350$, matrix $320 \times 240$; 
average $1 \mathrm{~mm}$ and axial Short Tau Inversion Recovery (STIR); TR/TE 4550/91; slice thickness $5 \mathrm{~mm}$ with $1 \mathrm{~mm}$ interslice gap, FOV $350 \times 350$, matrix $384 \times 269$; average $1 \mathrm{~mm}$ ) sequences were obtained.

The ADC maps were constructed from DWI images by using the mono-exponential model. Calculated and acquired high $b$-value DWI images were compared for lesion visibility and image quality by a radiologist with 5 years' experience in prostate with knowledge of the pathology data. Signal intensities on the high $b$-value $\left(1400 \mathrm{~s} / \mathrm{mm}^{2}\right)$ images of the peripheral zone, and the defined lesions were visualized. The exponential exp-ADC, sel-ADC, and ADCr of defined lesions were evaluated using region of interest-based measurements. The exp-ADC value calculated for the suspected lesion by drawing a region of interest (ROI) around the suspicious lesion using a ROI covering the whole of the lesion. Sel-ADC was measured, using a region of interest (ROI) of $5-10 \mathrm{~mm}^{2}$ at the area of lowest ADC value within the lesion. ADCr was calculated as ADC tumour/ADC mirror normal-appearing prostate parenchyma. Exp-ADC, sel-ADC, and ADCr were correlated with the Gleason score obtained via transrectal ultrasound-guided biopsy. The cases were classified into 2 groups, according to their Gleason score, as group I with Gleason score 6, and Gleason score 7-10 stratified as Group II.

Each biopsy was performed in a left lateral decubitus position. We used endocavitary 4 to $10 \mathrm{MHz}$ broadband curved array endfire transducer with Logiq E9 (General Electric Healthcare) and an 18-20 gauge cutting core biopsy needle. Patients first underwent systematic 14-core biopsies (6 from each lobe and 1 more from each transitional zone), followed by targeted biopsies generally consisting of 2 or 3 cores from each target.

\section{Histopathology analysis}

A genitourinary pathologist with 20 years' experience reviewed and described all biopsy cores. The specimens were stained with haematoxylin-eosin under a light microscope (Olympus BX51, Olympus Corporation, Tokyo 163-0914, Japan). The tumours from all patients were classified into Gleason score.

Table 1. Relationship between parameters and Gleason scores

\begin{tabular}{|l|c|c|c|c|}
\hline Parameter & Mean SD & $\begin{array}{c}\text { Gleason } \\
\text { score }=6\end{array}$ & \multicolumn{2}{|c|}{$\begin{array}{c}\text { Gleason score } \\
\geq 3+4\end{array}$} \\
\hline Age & $61.02 \pm 6.08$ & 62.04 & 60.8 & 0.27 \\
\hline PSA & $6.34 \pm 2.56$ & 4.34 & 7.02 & 0.6 \\
\hline PSA density & $0.16 \pm 0.09$ & 0.14 & 0.18 & 0.015 \\
\hline Exp-ADC & $772.93 \pm 180.11$ & 862.44 & 723.55 & 0.014 \\
\hline Sel-ADC & $639.64 \pm 162.94$ & 718.63 & 596.07 & 0.012 \\
\hline ADCr & $6.34 \pm 2.56$ & 0.64 & 0.54 & 0.09 \\
\hline
\end{tabular}

The biopsy data included the number of positive biopsy cores, Gleason score, and the maximal percentage of involvement for each positive biopsy core.

\section{Statistical analysis}

All statistical analyses were carried out using Windows for SPSS v. 22 (IBM Corporation, New York, USA). The mean sel-ADC and exp-ADC and ADCr values were compared between 2 groups. PSA and PSA density were assessed if there was a statistically significant difference between 2 groups. PSA and PSA density were assessed if there was a statistically significant difference between 2 groups.

Continuous variables were assessed using the ShapiroWilks test for normality of data and presented as mean \pm SD or median (range). The Mann-Whitney $U$ test or Student's $t$-test were performed to compare non-parametric and parametric variables. Also, receiver operating characteristic (ROC) curve analysis and areas under the curve (AUCs) were performed for each statistically significant parameter to establish an optimal cut-off value and to assess the discrimination ability of each parameter for nonsignificant Pca versus clinically significant PCa. The best cut-off point was determined by using the Youden $S$ index. Also, parameters were compared between the study and control groups with independent samples $t$-test.

Spearman's rank correlation analysis was used to show the correlations of aggressiveness of prostate $\mathrm{Ca}$ with sel-ADC, exp-ADC, and ADCr values. $P<0.05$ was considered statistically significant.

\section{Results}

A total of 69 patients were enrolled in this study. Fortyfour patients with Pca formed the study group, and 25 patients were observed to have no Pca with mpMRI. The median patient age in the study group was $61.02 \pm 6.08$ years (range, 50 to 84 years). The mean PSA was $6.34 \pm 2.56 \mathrm{ng} /$ $\mathrm{ml}$, and the median PSA Density was $0.16 \pm 0.09$. With respect to histopathological analysis, we identified 17 patients with a GS of $6 ; 20$ patients with a GS of $7 ; 2$ patients with a GS of 8; 4 patients with a GS of 9; and 1 patient with a GS of 10. Eleven patients had multifocal Pca in the study group. The GS of multifocal Pca group was 7 in 6 patients, and all of the patients with GS 8, 9, and 10 had multifocal Pca.

There was a nonsignificant correlation between PSA level and Gleason score $(p=0.6)$, but there was a significant difference between the 2 groups in terms of PSA density $(p=0.015)$. The ADCr was not significant between 2 groups, while sel-ADC and exp-ADC were statistically significant ( $p=0.09,0.014,0.012$, respectively) (Table 1 ).

The mean exp-ADC and sel-ADC values were significantly lower in high-grade Pca than in low-grade Pca ( $p=0.012$ and 0.014 , respectively). However, the mean ADCr value difference between nsPca and sPca was not significant $(p=0.09)$. 
ROC analysis was performed to obtain an optimal cutoff value for the sel-ADC and exp-ADC value to differentiate 2 groups (area under the curve $=0.719$ and 0.726 , respectively) (Figure 1). A sel-ADC value $\leq 684 \mathrm{~mm}^{2} / \mathrm{s}$ has a sensitivity, specificity, positive predictive value, negative predictive value, of 79.31, 62.50, 79.3, and 62.5 respectively, to differentiate nsPca from sPca. An exp-ADC value $\leq 731 \mathrm{~mm}^{2} / \mathrm{s}$ has a sensitivity, specificity, positive predictive value, and negative predictive value of 55.17, 87.50, 69 , and 56.3, respectively, to differentiate nsPca from sPca.

The AUC of exp-ADC was 0.726, AUCs of sel-ADC were 0.719 and $\mathrm{ADCr}$ was 0.702 , respectively (Figure 1). The exp-ADC values were thus strongly associated with higher tumour aggressiveness in this study.

The sel-ADC value was $0.718 \pm 0.161 \times 10^{-3} \mathrm{~mm}^{2} / \mathrm{s}$ for Group I; $0.596 \pm 0.148 \times 10^{-3} \mathrm{~mm}^{2} / \mathrm{s}$ for Group II; the exp-ADC was $0.862 \pm 0.188 \times 10^{-3} \mathrm{~mm}^{2} / \mathrm{s}$ for Group I; and $0.723 \pm 0.157 \times 10^{-3} \mathrm{~mm}^{2} / \mathrm{s}$ for Group II, the ADCr was $0.64 \pm 0.2$ for Group I and $0.54 \pm 0.15$ for Group II.

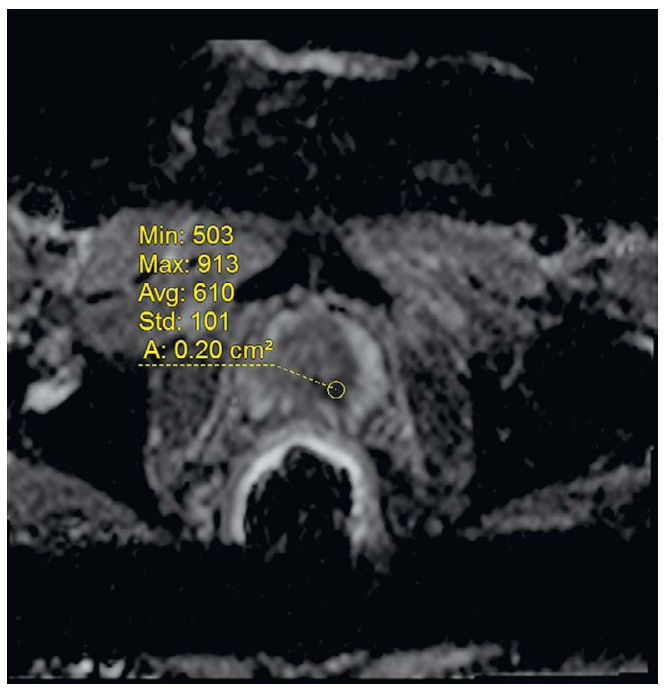

Figure 2. A 67-year-old patient with Gleason Score $3+4$ prostate adenocarcinoma in the left peripheral zone, exp-ADC value is measured

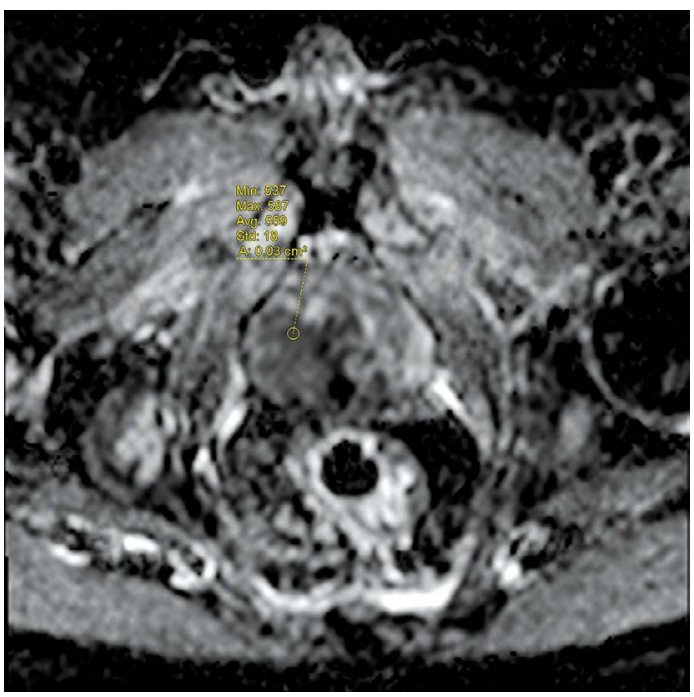

Figure 3. A 67-year-old patient with Gleason Score $3+4$ prostate adenocarcinoma in the left peripheral zone, sel-ADC value is measured

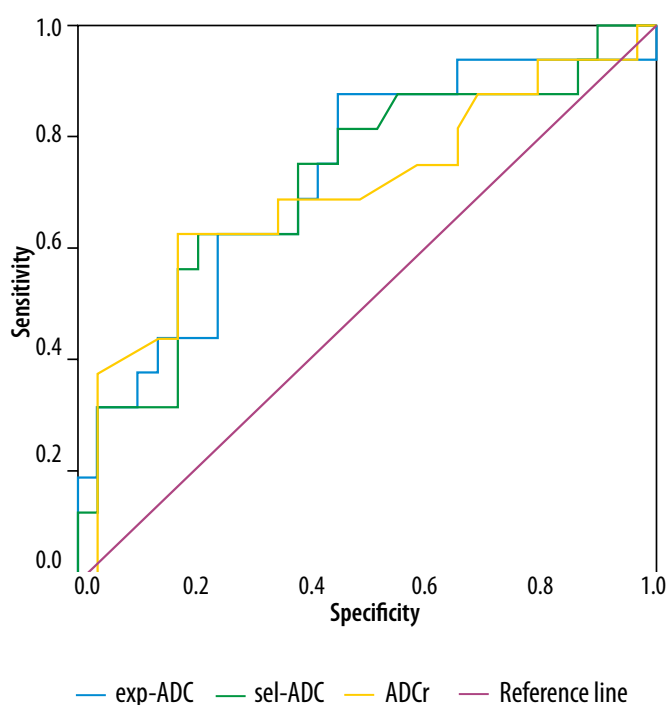

Figure 1. Area under the curve (AUC) of exp-ADC was 0.726 , AUCs of sel-ADC were 0.719 , and $A D C r$ was 0.702

Representative cases with a GS of $3+4$ and $4+4$ prostate cancers in the right and left peripheral zones are shown in Figures 2-4 and Figures 5-8, respectively. The differences between the ADC values obtained for 2 groups were analysed in pairs, and there were significant differences between GS 6 groups with the exception of GS $\geq 3+4$. Furthermore, the sel-ADC, exp-ADC, and ADCr values of tumours with GS $\geq 3+4$ were significantly lower than that of tumours with GS $=6(p<0.001)$ (Table 1$)$.

The relation between age, PSA, PSA density, exp-ADC, sel-ADC, prostate MR volume, and prostate adenocarcinoma is shown in Table 2. ADCr could not be studied in the control group because there was no tumoural prostatic tissue in the control group.

The age, PSA, PSA density, and prostate MR volume were at statistically significantly higher levels in patients with Pca than in the patients without pCa $(p<0.01)$. Exp-

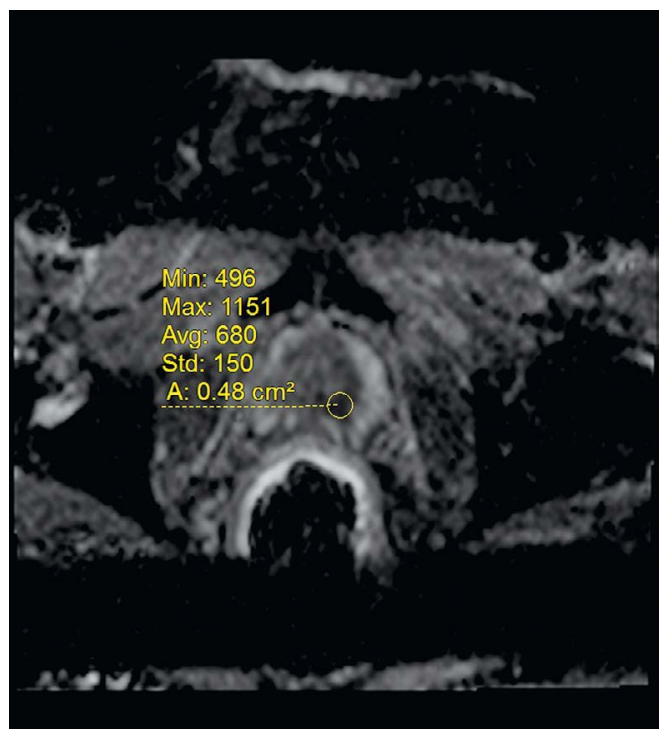

Figure 4. T2-weighted axial image of the same patient 


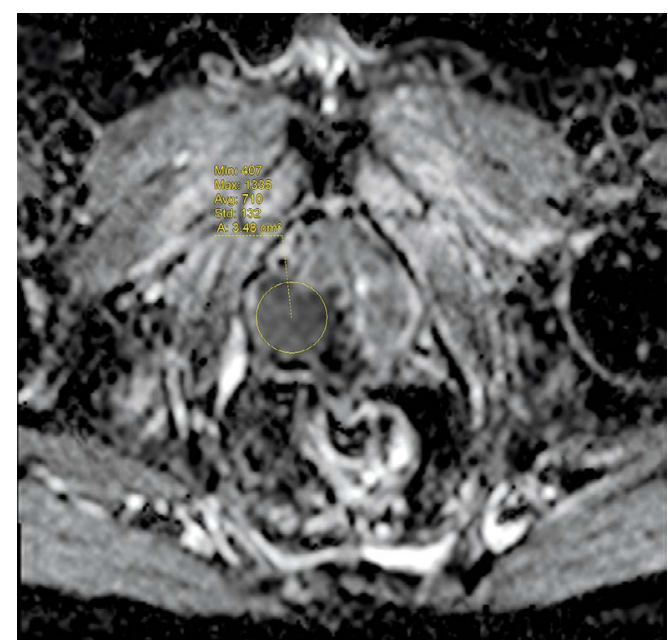

Figure 5. A 62-year-old patient with Gleason score $4+4$ in the right peripheral zone on diffusion-weighted imaging, sel-ADC value is measured

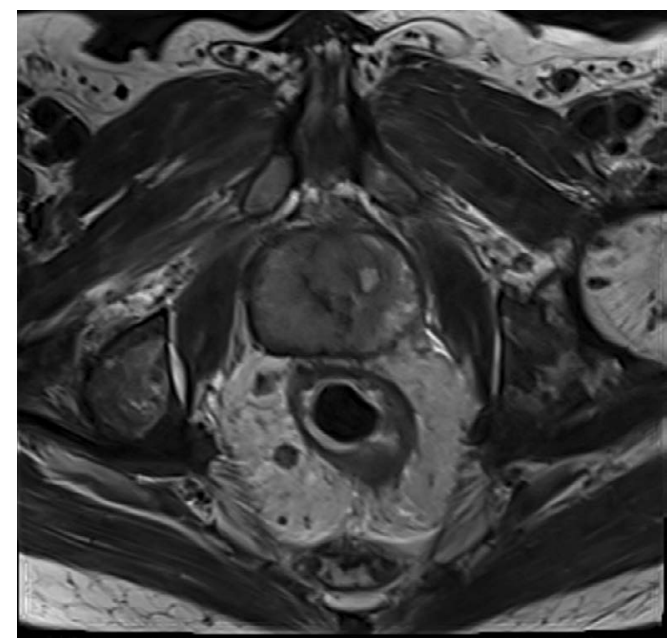

Figure 7. T2-weighted image of a patient with right peripheral Gleason score $4+4$ prostate adenocarcinoma

ADC and sel-ADC values were at statistically significantly lower levels in patients with Pca than in the cases without Pca $(p<0.01)$.

\section{Discussion}

MRI can delineate the structure of prostate and anatomical details of the prostatic tissue. DWI, a major component

Table 2. Relation between parameters and prostate adenocarcinoma

\begin{tabular}{|l|c|c|c|}
\hline Parameter & $\begin{array}{c}\text { PCa+ } \\
\text { Mean SD }\end{array}$ & $\begin{array}{c}\text { PCa- } \\
\text { Mean SD }\end{array}$ & $P$ \\
\hline Age & $61.02 \pm 6.08$ & $53.84 \pm 12.01$ & $0.001^{* a}$ \\
\hline PSA & $6.34 \pm 2.56$ & $0.96 \pm 0.58$ & $0.001^{* a}$ \\
\hline PSA density & $0.16 \pm 0.09$ & $0.02 \pm 0.01$ & $0.001^{* a}$ \\
\hline Ext-ADC & $772.93 \pm 180.11$ & $1570.80 \pm 245.29$ & $0.001^{* a}$ \\
\hline Sel-ADC & $639.64 \pm 162.94$ & $1540.08 \pm 234.56$ & $0.001^{* a}$ \\
\hline Prostate MR volume & $46.46 \pm 19.94$ & $38.36 \pm 19.34$ & $0.001^{* a}$ \\
\hline
\end{tabular}

Independent samples $t$-test, ${ }^{*} p<0.01$

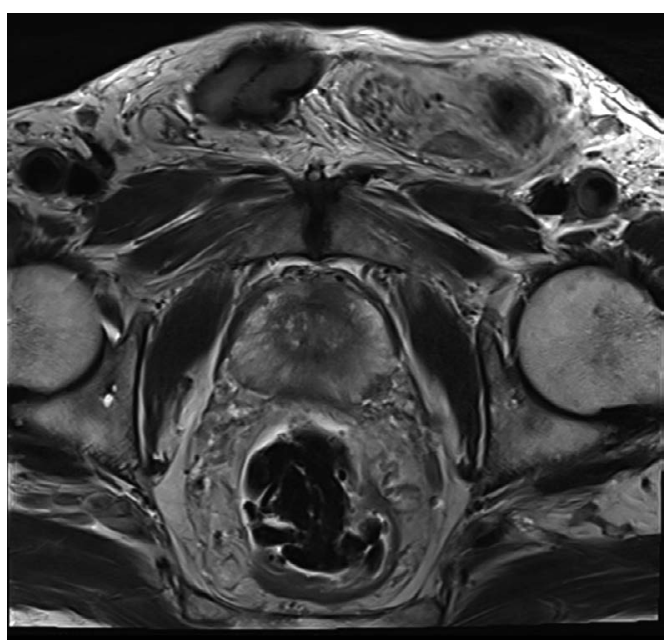

Figure 6. Exp-ADC value of a patient with right peripheral Gleason score $4+4$ prostate adenocarcinoma

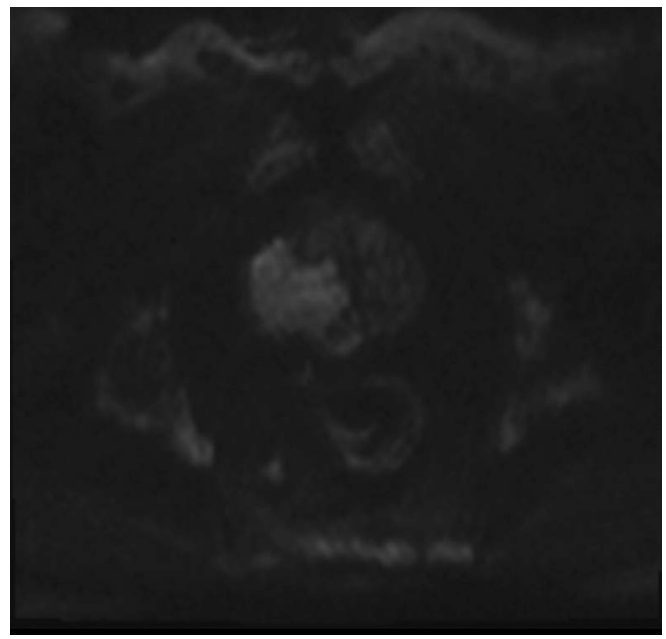

Figure 8. Computed $b$ value of this patient

of mpMRI, has been the main sequence for detecting and staging prostate cancer in $\mathrm{PZ}$, and it additionally provides information about tumour cellularity. In particular, DWI and ADC values are regarded as reliable non-invasive tools for assessing tumour aggressiveness in prostate cancer [18]. MRI can be a guide for prostate biopsies or demonstrates the anatomic location of the tumour and is a follow-up method for active surveillance of patients. Many studies have been performed on the discrimination between normal and malignant prostate tissue using the DWI technique. Because absolute $\mathrm{ADC}$ values depend on the selected $b$-value, the most accurate method of measuring the ADC value is still being discussed. Cellularity, tumour type, or nuclear grade can affect the ADC values of tumoural tissue.

Therefore, different options are under investigation beyond ADC tumour mean value, such as the sel-ADC value, exp-ADC, and ADCr (expressed as the ratio between tumour and non-tumour ADC values) in prostate cancer aggressiveness.

Hambrock et al. [19] showed that a significant inverse correlation between ADC values and Gleason score was 
well-illustrated. Some studies have reported that ADC values have demonstrated significant overlaps within the same GS groups $[10,11]$. MRI acquisition parameters, selected $b$ values, patient properties, and prostate gland structure cause differences in the obtained ADC values. To eliminate the effect of these parameters, this study assessed the role of sel-ADC, exp-ADC, and the ADCr in distinguishing Gleason score 6 and Gleason Score $\geq 3+4$. Our study demonstrated that exp-ADC values had highest specificity with $87.5 \%$ inversely correlated with GS in prostate cancer, and that it was feasible to discriminate between pathologic GS 6 diseases according to ADC values.

In our study there were significant differences in sel$\mathrm{ADC}$ and exp-ADC when comparing 2 groups.

Wu et al. [20] showed that higher ADC values $(0.830$ $\times 10^{-3} \mathrm{~mm}^{2} / \mathrm{s}$ ) were significantly associated with low-risk prostate cancer (GS 6 disease).

In our study, the cut-off values for sel-ADC and expADC were $0.684 \times 10^{-3} \mathrm{~mm}^{2} / \mathrm{s}$ and $0.731 \times 10^{-3} \mathrm{~mm}^{2} / \mathrm{s}$, respectively, for differentiating low-risk prostate cancer from high-risk prostate cancer.

The decrease in ADC in high GS disease can be explained by the high tumour cellularity representing tumour aggressiveness.

Many authors affirm that the ADC ratio seems to be a more useful and reproducible method because it is independent of the diffusion parameters as selected $b$ values and vector strength [21-24]. Nevertheless, a number of factors still limit ADCr's efficacy. In our opinion, prostate tissue without tumour involvement used to measure ADCr appears norma, but it contains benign conditions like prostatitis, hyperplasia, or fibrosis.
When all these factors are taken into consideration, in our opinion sel-ADC and exp-ADC measurements are safe methods for prostate cancer aggressiveness assessment.

One limitation of this study might be the limited number of patients. Secondly, we compared our MRI parameters with GS. Sometimes GS on a biopsy does not reflect the cancer's true grade. Additionally, recent modifications have improved the Gleason grading system, but it is still much more complex and can be confusing for patients and clinicians. For example, Gleason score 7 includes both Gleason score $3+4$ and $4+3$. Many recent studies have shown that Gleason score $4+3=7$ demonstrates worse pathological stage than $3+4=7$, although many clinicians consider Gleason score 7 on biopsy to be intermediate risk [25-27].

\section{Conclusions}

Sel-ADC and exp-ADC are more useful diffusion parameters than $\mathrm{ADCr}$ for proving prostate cancer aggressiveness. Exp-ADC and sel-ADC measurements in diffusion imaging improved specificity, accuracy, and AUC for detecting clinically relevant prostate carcinoma.

Our study results show that they are promising parameters to identify clinically significant prostate adenocarcinoma.

\section{Conflict of interest}

The author reports no conflict of interest.

\section{References}

1. Siegel RL, Miller KD, Jemal A. Cancer statistics. CA Cancer J Clin 2017; 67: 7-30.

2. American College of Radiology. MR prostate imaging reporting and data system version 2.0. Reston, VA: American College of Radiology. Available at: www.acr.org/Quality-Safety/Resources/PIRADS/ (Accessed: 12.12.2015).

3. Ahmed HU, Akin O, Coleman JA, et al. Transatlantic Consensus Group on active surveillance and focal therapy for prostate cancer. BJU Int 2012; 109: 1636e47.

4. de Rooij M, Hamoen EH, Futterer JJ, et al. Accuracy of multiparametric MRI for prostate cancer detection: a meta-analysis. AJR Am J Roentgenol 2014; 202: 343-351.

5. Barentsz JO, Richenberg J, Clements R, et al. ESUR prostate MR guidelines 2012. Eur Radiol 2012; 22: 746-757.

6. Hambrock T, Somford DM, Hoeks C, et al. Magnetic resonance imaging guided prostate biopsy in men with repeat negative biopsies and increased prostate specific antigen. J Urol 2010; 183: 520-528.

7. Hoeks CM, Hambrock T, Yakar D, et al. Transition zone prostate cancer: detection and localization with 3-T multiparametric MR imaging. Radiology 2013; 266: 207-217.
8. Selnaes KM, Heerschap A, Jensen LR, et al. Peripheral zone prostate cancer localization by multiparametric magnetic resonance at $3 \mathrm{~T}$ : unbiased cancer identification by matching to histopathology. Invest Radiol 2012; 47: 624-633.

9. Delongchamps NB, Rouanne M, Flam T, et al. Multiparametric magnetic resonance imaging for the detection and localization of prostate cancer: combination of $\mathrm{T} 2$-weighted, dynamic contrastenhanced, and diffusion-weighted imaging. BJU Int 2011; 107: 1411-1418.

10. Dickinson L, Ahmed HU, Allen C, et al. Magnetic resonance imaging for the detection, localisation, and characterisation of prostate cancer: recommendations from a European consensus meeting. Eur Urol 2011; 59: 477-494.

11. Pereira FP, Martins G, Figueiredo E, et al. Assessment of breast lesions with diffusion-weighted MRI: Comparing the use of different b values. AJR Am J Roentgenol 2009; 193: 1030-1035.

12. Park SY, Kim CK, Park BK, et al. Prediction of biochemical recurrence following radical prostatectomy in men with prostate cancer by diffusion-weighted magnetic resonance imaging: Initial results. Eur Radiol 2011; 21: 1111-1118. 
13. Hauth E, Halbritter D, Jaeger H, et al. Diagnostic value of semiquantitative and quantitative analysis of functional parameters in multiparametric MRI of the prostate. Br J Radiol 2017; 90: 20170067.

14. Wu X, Reinikainen P, Vanhanen A, et al. Correlation between apparent diffusion coefficient value on diffusion-weighted MR imaging and Gleason score in prostate cancer. Diagn Interv Imaging 2017; 98: 63-71.

15. Epstein JI, Allsbrook WC Jr, Amin MB, Egevad LL; ISUP Grading Committee. The 2005 International Society of Urological Pathology (ISUP) Consensus Conference on Gleason Grading of Prostatic Carcinoma. Am J Surg Pathol 2005; 29: 1228-1242.

16. Vargas HA, Akin O, Franiel T, et al. Difusion-weighted endorectal $\mathrm{MR}$ imaging at $3 \mathrm{~T}$ for prostate cancer: tumor detection and assessment of aggressiveness. Radiology 2011; 259: 775-784.

17. Turkbey B, Shah VP, Pang Y, et al. Is apparent diffusion coefficient associated with clinical risk scores for prostate cancers that are visible on 3-T MR images? Radiology 2011; 258: 488-495.

18. Jie $\mathrm{C}$, Rongbo $\mathrm{L}$, Ping $\mathrm{T}$. The value of diffusion-weighted imaging in the detection of prostate cancer: a meta-analysis. Eur Radiol 2014; 24: 1929-1941.

19. Hambrock T, Somford DM, Huisman HJ, et al. Relationship between apparent diffusion coefficients at 3.0-T MR imaging and Gleason grade in peripheral zone prostate cancer. Radiology 2011; 259: 453461.

20. Wu X, Reinikainen P, Vanhanen A, et al. Correlation between apparent diffusion coefficient value on diffusion-weighted MR imaging and Gleason score in prostate cancer. Diagnostic Intl Imaging 2017; 98: 63-71.

21. Boesen L, Chabanova E, Løgager V, et al. Apparent diffusion coefficient ratio correlates significantly with prostate cancer gleason score at final pathology. J Magn Reson Imaging 2015; 42: 446-453.

22. Lebovici A, Sfrangeu SA, Feier D, et al. Evaluation of the normal-to-diseased apparent diffusion coefficient ratio as an indicator of prostate cancer aggressiveness. BMC Med Imaging 2014; 14: 15.

23. De Cobelli F, Ravelli S, Esposito A, et al. Apparent diffusion coefficient value and ratio as noninvasive potential biomarkers to predict prostate cancer grading: Comparison with prostate biopsy and radical prostatectomy specimen. AJR Am J Roentgenol 2015; 204: 550-557.

24. Alessandrino F, Taghipour M, Hassanzadeh E, et al. Predictive role of PI-RADSv2 and ADC parameters in differentiating Gleason pattern $3+4$ and $4+3$ prostate cancer. Abdom Radiol (NY) 2019; 44: 279-285.

25. Burdick MJ, Reddy CA, Ulchaker J, et al. Comparison of biochemical relapse-free survival between primary Gleason score 3 and primary Gleason score 4 for biopsy Gleason score 7 prostate cancer. Int J Radiat Oncol Biol Phys 2009; 73: 1439-1445.

26. Chan TY, Partin AW, Walsh PC, Epstein JI. Prognostic significance of Gleason score 3+4 versus Gleason score $4+3$ tumor at radical prostatectomy. Urology 2000; 56: 823-827.

27. Kang DE, Fitzsimons NJ, Presti JC Jr, et al. Risk stratification of men with Gleason score 7 to 10 tumors by primary and secondary Gleason score: results from the SEARCH database. Urology 2007; 70: 277-282. 